\title{
THYROID
}

\section{Laser ablation of thyroid nodules is rapid, safe and effective}

Laser ablation therapy (LAT) is an outpatient procedure used to treat thyroid nodules. Although many small prospective trials have been completed, no real-world data have been reported that describe the effectiveness of the procedure or the associated risks of complications.

In a new retrospective study from eight centres in Italy, ultrasound-guided LAT of benign thyroid nodules was assessed in 1,531 patients. Patients were evaluated immediately after the LAT session, as well as at $24 \mathrm{~h}$, within the following 30 days and finally at 12 months.

Overall, $83 \%$ of nodules were treated with a single LAT session, the remaining patients required up to three treatments. At 12 months, the mean reduction in the volume of thyroid nodules was $72 \pm 11 \%$ (ranging from $48 \%$ to $96 \%$ ). In total, only 17 complications $(0.9 \%)$ were recorded and all were reported in the first $24 \mathrm{~h}$ after the procedure. Of the major complications, eight patients experienced voice changes; however, these symptoms resolved within
2-84 days with corticosteroid treatment. Similarly, only nine patients reported minor complications, which were perithyroid haematoma $(n=8)$ and skin burn $(n=1)$; however, this burn was an isolated procedural accident. Patient reports of mild, moderate and severe pain were also low $(3.3 \%, 1.9 \%$ and $0.4 \%$, respectively).

Interestingly, patients treated without local anaesthetic reported less pain than those who received local anaesthetic. The investigators highlight that pain during the procedure enables a clinician to reposition the LAT apparatus, which might reduce the risk of procedural complications, and note that real-world experience suggests LAT is an effective and rapid outpatient procedure.

\section{Tim Geach}

Original article Pacella, C. M. et al. Outcomes and risk factors for complications of laser ablation for thyroid nodules. A multicenter study on 1531 patients. J. Clin. Endocrinol. Metab. doi:10.1210/jc.2015-1964 